The Astrophysical Journal, 615:344-353, 2004 November 1

(C) 2004. The American Astronomical Society. All rights reserved. Printed in U.S.A.

\title{
METHANE ABUNDANCE VARIATIONS TOWARD THE MASSIVE PROTOSTAR NGC 7538 IRS $9^{1,2}$
}

\author{
A. C. A. Boogert ${ }^{3}$ G. A. Blake, ${ }^{4,5}$ And K. ÖBerG ${ }^{5}$ \\ Received 2004 March 4; accepted 2004 July 8
}

\begin{abstract}
Absorption and emission lines originating from the $\nu_{3} \mathrm{C}-\mathrm{H}$ stretching manifold of gas-phase $\mathrm{CH}_{4} \mathrm{were}$ discovered in the high-resolution $(R=25,000)$ infrared $L$-band spectrum along the line of sight toward NGC 7538 IRS 9. These observations provide a diagnostic of the complex dynamics and chemistry in a massive star-forming region. The line shapes resemble P Cygni profiles with the absorption and emission components shifted by $\sim 7 \mathrm{~km}$ $\mathrm{s}^{-1}$ with respect to the systemic velocity. Similar velocity components were observed in $\mathrm{CO}$ at $4.7 \mu \mathrm{m}$, but in contrast to $\mathrm{CH}_{4}$, the $\mathrm{CO}$ shows deep absorption due to a high-velocity outflow as well as absorption at the systemic velocity due to the cold outer envelope. It is concluded that the gas-phase $\mathrm{CH}_{4}$ abundance varies by an order of magnitude in this line of sight: it is low in the envelope and the outflow $\left(X\left[\mathrm{CH}_{4}\right]<0.4 \times 10^{-6}\right)$ and at least a factor of 10 larger in the central core. The discovery of solid $\mathrm{CH}_{4}$ in independent ground- and space-based data sets shows that methane is nearly entirely frozen onto grains in the envelope. It thus appears that $\mathrm{CH}_{4}$ is formed by grain surface reactions, evaporates into the gas phase in the warm inner regions of protostellar cores, and is efficiently destroyed in shocks related to outflows.
\end{abstract}

Subject headings: astrochemistry — infrared: ISM — ISM: abundances — ISM: molecules — stars: formation — stars: individual (NGC 7538 IRS 9)

\section{INTRODUCTION}

Among the suite of molecules observed in interstellar and circumprotostellar media, ${ }^{6}$ methane is relatively poorly studied. $\mathrm{CH}_{4}$ has no permanent dipole moment and therefore cannot be observed by pure rotational transitions at radio wavelengths. Its strongest fundamental $(v=1-0)$ rovibrational transitions, i.e., the $\nu_{3} \mathrm{C}-\mathrm{H}$ stretching and the $\nu_{4} \mathrm{C}-\mathrm{H}$ bending modes, occur at 3.32 and $7.67 \mu \mathrm{m}$, respectively. Strong telluric absorption at these wavelengths hinders interstellar $\mathrm{CH}_{4}$ studies. Gas- and solid-phase $\mathrm{CH}_{4}$ have been positively identified at $7.67 \mu \mathrm{m}$ in three sight lines: the massive protostars NGC 7388 IRS 9, W33A, and GL 7009 S (Lacy et al. 1991; Boogert et al. 1996, 1997, 1998; Dartois et al. 1998). $\mathrm{CH}_{4}$ was detected only in the solid state in low-resolution Infrared Space Observatory (ISO) observations at $7.67 \mu \mathrm{m}$ toward $\sim 30$ low-mass protostars (White et al. 2000; Gürtler et al. 2002; Alexander et al. 2003), the Galactic center (Chiar et al. 2000), and an external galactic nucleus (Spoon et al. 2001). A recent low-resolution Spitzer Space Telescope spectrum shows absorption by solid $\mathrm{CH}_{4}$ toward the young, low-mass protostar IRAS $08242+5100$ (NoriegaCrespo et al. 2004; Boogert et al. 2004).

Observations of $\mathrm{CH}_{4}$ provide insight into the basic principles of astrochemical networks. In the presence of atomic $\mathrm{C}$ and $\mathrm{H}$, $\mathrm{CH}_{4}$ is rapidly formed on cold grains, just as $\mathrm{H}_{2} \mathrm{O}$ is formed

\footnotetext{
${ }^{1}$ Some of the data presented herein were obtained at the W. M. Keck Observatory, which is operated as a scientific partnership among the California Institute of Technology, the University of California, and the National Aeronautics and Space Administration. The Observatory was made possible by the generous financial support of the W. M. Keck Foundation.

${ }^{2}$ Based on observations with ISO, an ESA project with instruments funded by ESA Member States (especially the PI countries: France, Germany, the Netherlands, and the United Kingdom), and with the participation of ISAS and NASA.

3 California Institute of Technology, Division of Physics, Mathematics, and Astronomy, 105-24, Pasadena, CA 91125; acab@astro.caltech.edu.

4 California Institute of Technology, Division of Geological and Planetary Sciences, 150-21, Pasadena, CA 91125.

${ }^{5}$ California Institute of Technology, Division of Chemistry and Chemical Engineering, Pasadena, CA 91125.

${ }^{6}$ See the compilation at http://www.cv.nrao.edu/ awootten/allmols.html.
}

from atomic $\mathrm{O}$ and $\mathrm{H}$ (e.g., Brown et al. 1988). Perhaps surprisingly, the observed gas- and solid-state $\mathrm{CH}_{4}$ abundances are low, not more than a few percent with respect to $\mathrm{CO}$ and $\mathrm{H}_{2} \mathrm{O}$ (Lacy et al. 1991; Boogert et al. 1998). This points to relatively low atomic $\mathrm{C}$ abundances at the time of $\mathrm{CH}_{4}$ formation, when most $\mathrm{C}$ is already locked up in $\mathrm{CO}$. In this same scenario the high $\mathrm{CH}_{3} \mathrm{OH}$ abundances in several lines of sight may be explained by hydrogenation of abundantly present $\mathrm{CO}$ (Dartois et al. 1999; Pontoppidan et al. 2003), and large $\mathrm{CO}_{2}$ abundances may be explained by oxidation of $\mathrm{CO}$. The dominance of a COand O-based, rather than C-based, chemistry is stressed by the profiles of solid $\mathrm{CO}_{2}$ absorption bands, which show an intimate mixture of $\mathrm{CO}_{2}, \mathrm{CH}_{3} \mathrm{OH}$, and $\mathrm{H}_{2} \mathrm{O}$ in the ices (Gerakines et al. 1999; Boogert et al. 2000). Nevertheless, even at relatively low abundances methane is proposed to be the starting point of a rich chemistry leading to the complex organic molecules observed in dense clouds like the TMC-1 ridge (Markwick et al. 2000). To further test the role of $\mathrm{CH}_{4}$ in interstellar chemistry, basic diagnostics, such as gas-phase abundances, gas-phase/ solid-phase abundance ratios, gas-phase temperatures, and abundance gradients need to be measured in more lines of sight.

In this work we report the discovery of absorption and emission features arising from the $3.32 \mu \mathrm{m} \mathrm{C}-\mathrm{H}$ stretching mode of gas- and solid-phase $\mathrm{CH}_{4}$. A previous attempt to detect $\mathrm{CH}_{4}$ at this wavelength was unsuccessful and indicated an abundance of less than $10^{-6}$ with respect to $\mathrm{H}$ in the Orion $\mathrm{BN}$ object (Knacke et al. 1985). We study the line of sight toward the massive protostar NGC 7538 IRS 9, because its high radial velocity of $V_{\mathrm{lsr}}=-57 \mathrm{~km} \mathrm{~s}^{-1}$ (e.g., van der Tak et al. 2000) facilitates the separation of telluric and interstellar lines. NGC 7538 IRS 9 is a well-studied massive protostar, known for its large ice and gas columns (Whittet et al. 1996; Mitchell et al. 1990) and a prominent molecular outflow (Mitchell et al. 1991). The $R(0)$ and $R(2)$ lines of the bending mode of gas-phase $\mathrm{CH}_{4}$ and solid-state $\mathrm{CH}_{4}$ were detected in this source by Lacy et al. (1991). Unambiguous confirmations of these detections were obtained with space-based observations (Boogert et al. 1996, 1998). Here we independently confirm the presence of $\mathrm{CH}_{4}$ by detecting many lines from the $3.32 \mu \mathrm{m}$ stretching mode as well 
as the ice band. The high spectral resolution of these observations permits a line profile analysis in which we determine gasphase conditions in the various environments along the line of sight, such as the envelope, the hot core, and the outflow. This in turn elucidates the formation and destruction pathways of $\mathrm{CH}_{4}$ in these different environments.

The Keck/NIRSPEC and ISO SWS observations are discussed in $\S 2$ and presented in $\S 3.1$. A profile analysis of the gas-phase absorption and emission $\mathrm{CH}_{4}$ lines, in conjunction with previously reported high-resolution $\mathrm{CO}$ observations, is presented in $\S \S 3.2 .1$ and 3.2.2. The ice band profile is discussed and compared with the $\mathrm{C}-\mathrm{H}$ bending mode in $\S 3.3$. The implications of these results on the formation and evolution history of the $\mathrm{CH}_{4}$ molecule in protostellar environments are discussed in $\S 4.1$. The origin of the low-velocity, warm gas, responsible for the P Cygni-like line profiles, is further constrained in $\S 4.2$. We conclude with future prospects in $\S 5$.

\section{OBSERVATIONS}

\subsection{Keck/NIRSPEC $R=25,000$ Spectroscopy}

The high-resolution $L$-band spectrum of the massive protostar NGC 7538 IRS 9 was obtained with the NIRSPEC spectrometer (McLean et al. 1998) at the Keck II telescope atop Mauna Kea. Observations were made on five different nights with clear and dry weather conditions: UT 2002 December 17 and 18, 2003 July 10 and 11, and 2003 November 2. NIRSPEC was used in the echelle mode with the 0 ". $43 \times 24^{\prime \prime}$ slit, providing a resolving power of $R=\lambda / \Delta \lambda=25,000\left(\sim 12 \mathrm{~km} \mathrm{~s}^{-1}\right)$. The $2.9-4.1 \mu \mathrm{m}$ region was almost fully covered in five echelle settings, each covering five Nyquist-sampled wavelength orders of $\sim 0.05 \mu \mathrm{m}$ on the $1024^{2}$ pixel array. Two small regions centered on 2.97 and $3.12 \mu \mathrm{m}$ were not covered. The on-source integration time per setting was 20 minutes. Several $\mathrm{CH}_{4}$ rovibrational lines, as well as the solid $\mathrm{CH}_{4}$ band at $3.32 \mu \mathrm{m}$, were observed multiple times to verify their reality. At $V_{\mathrm{lsr}}=$ $-57 \mathrm{~km} \mathrm{~s}^{-1}$ for NGC 7538 IRS 9, the Doppler velocity with respect to Earth was $-53.5,-82.4$, and $-64.0 \mathrm{~km} \mathrm{~s}^{-1}$ for the three observing runs. This proved to be sufficient to separate interstellar and telluric $\mathrm{CH}_{4}$ lines and guarantees their reality. The spectra presented in this paper are an average of all the data. The data were reduced in a standard way, using IDL routines (Boogert et al. 2002). Atmospheric absorption features were divided out using the standard star HR 8585 (A1 V), which is bright $(V=3.78)$ and reasonably close to NGC 7538 IRS 9 (an air mass of 1.20-1.30 vs. 1.34 for NGC 7538 IRS 9). The spectral shape and hydrogen absorption features in the standard star were divided out with a Kurucz model atmosphere. The hydrogen absorption profiles in the Kurucz model are accurate to $1 \%-2 \%$, and residuals can be seen near strong lines. This does not affect the region of main interest for this paper (3.15$3.65 \mu \mathrm{m}$ ), except perhaps near $3.297 \mu \mathrm{m}$, because of the $\mathrm{H}$ I $\operatorname{Pf} \delta$ line. Overall, a good telluric correction was achieved, resulting in signal-to-noise ratio $(\mathrm{S} / \mathrm{N})$ values of $\sim 110$ in the region of $\mathrm{CH}_{4}$ at $3.32 \mu \mathrm{m}, \sim 40$ at the bottom of the $\mathrm{H}_{2} \mathrm{O}$ ice band at $3.0 \mu \mathrm{m}$, and $\sim 200$ at wavelengths greater than $3.5 \mu \mathrm{m}$. Strong atmospheric lines, i.e., with less than $50 \%$ of the maximum transmission in each setting, leave residuals and were removed from the final spectrum. The spectra were wavelength-calibrated on the atmospheric emission lines, using an atmospheric model spectrum (P. van der Valk \& P. Roelfsema 1995, private communication). All orders were subsequently combined by applying relative multiplication factors. For the analysis of the emission lines $(\S 3.2 .1)$, the spectrum is flux-calibrated by scaling to the continuum brightness of the data presented in Whittet et al. (1996). Finally, in conjunction with the $\mathrm{CH}_{4}$ features, we analyze Keck/NIRSPEC $M$-band CO lines in NGC 7538 IRS 9, previously published in Boogert et al. (2002).

\subsection{ISO SWS $R=1500$ Spectroscopy}

The 3.26-3.38 $\mu \mathrm{m}$ spectral region of NGC 7538 IRS 9 was observed multiple times in the high-resolution $R=1500$ AOT06 mode of the Short Wavelength Spectrometer (SWS) on board ISO (Kessler et al. 1996; de Graauw et al. 1996). From the ISO archive we have selected the longest integrations in this wavelength range, corresponding to TDT numbers 39002336 (UT 1996 December 11), 75101049 (UT 1997 December 5), and 85200455 (UT 1998 March 16). All observations were part of the ICE_BAND/DWHITTET guaranteed time program and have not been previously published. The combined on-target integration time in the $3.26-3.38 \mu \mathrm{m}$ region amounts to $5219 \mathrm{~s}$. The individual data sets were processed with SWS Interactive Analysis and calibration files, version 10.1, in 2003 April at SRON/ Groningen. The three AAR products were combined, and the detector scans were aligned to the mean using second-order polynomials. Then, in this highly oversampled spectrum, data points deviating more than $3 \sigma$ from the mean per resolution element were removed. Finally, the data points were averaged and resampled to an $R=1500$ Nyquist-sampled grid. The resulting $\mathrm{S} / \mathrm{N}$ is 100 but significantly poorer at the outer $0.015 \mu \mathrm{m}$ edges.

\section{RESULTS}

\subsection{Features in the 3.1-3.6 $\mu \mathrm{m}$ Spectral Region}

The $L$-band spectrum of NGC 7538 IRS 9 is dominated by the deep absorption band of the $\mathrm{O}-\mathrm{H}$ stretching mode of $\mathrm{H}_{2} \mathrm{O}$ ice (Willner et al. 1982; Whittet et al. 1996). Its peak optical depth at $3.0 \mu \mathrm{m}$ is $\sim 4.5$. The most prominent substructure in the longwavelength wing of $\mathrm{H}_{2} \mathrm{O}$ is the well-known, yet unidentified, $3.47 \mu \mathrm{m}$ absorption band (Allamandola et al. 1992; Brooke et al. 1996; Dartois et al. 2002). We applied a smooth spline continuum to extract this band on an optical depth scale (Fig. 1). The results were verified by applying low-order polynomials to continuum regions described in previous studies (e.g., Brooke et al. 1999). The absorption shape is in good agreement with the lowresolution spectrum of Allamandola et al. (1992), as is the presence of a subband at $3.53 \mu \mathrm{m}$, attributed to the $\mathrm{C}-\mathrm{H}$ stretching mode of solid $\mathrm{CH}_{3} \mathrm{OH}$. A previously undetected absorption band is apparent by a downturn in the continuum slope at $3.32 \mu \mathrm{m}$. Although it is in a spectral region badly affected by the telluric $\mathrm{CH}_{4} Q$-branch, its presence is confirmed in Keck/NIRSPEC observations on different dates. In addition, a feature at the same wavelength is found in the space-based ISO SWS spectrum (Fig. 1). We thus conclude that this feature is real and is due to the stretching mode of solid $\mathrm{CH}_{4}(\S 3.3)$. Not immediately obvious in Figure 1 but clearly detected on close inspection (Fig. 2) are $P-, Q-$, and $R$-branch lines of gas-phase $\mathrm{CH}_{4}$. This is the first time that the $3.32 \mu \mathrm{m}$ ice and gas features have been detected in a circumprotostellar environment.

A number of additional features, not further discussed in this paper, are visible in the high-resolution Keck/NIRSPEC spectrum. Although they are reproduced in observations at different dates, they are entirely new and need to be confirmed in a larger sample of sources or, as for solid $\mathrm{CH}_{4}$, in independent data. A weak feature at $3.36 \mu$ m coincides with the $\mathrm{C}-\mathrm{H}$ stretch modes of ethanol (Allamandola et al. 1992) and ethane (Boudin et al. 1998). Interestingly, another band of ethane at $3.47 \mu \mathrm{m}$ seen in laboratory ices appears to be present in NGC 7538 IRS 9 as 


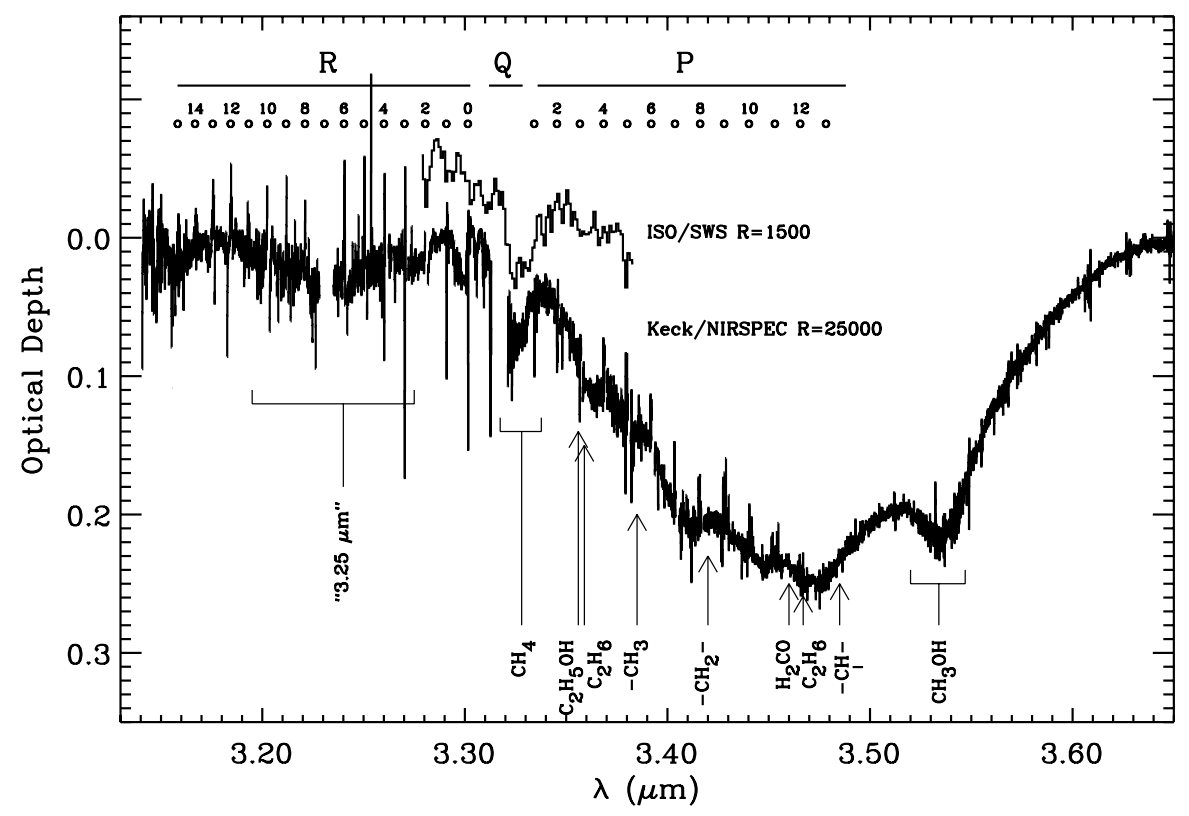

FIG. 1.-The $R=25,000 \mathrm{Keck} / \mathrm{NIRSPEC} L$-band spectrum of NGC 7538 IRS 9 on an optical depth scale, after correction for $\mathrm{H}_{2} \mathrm{O}$ ice absorption. The broad, deep absorption is the well-known, yet unidentified, " $3.47 \mu \mathrm{m}$ " absorption band. The presence of absorption by the $\mathrm{C}-\mathrm{H}$ stretching mode of solid $\mathrm{CH}_{4}$ is confirmed in the $R=1500 I S O$ SWS spectrum. $P_{-}, Q-$, and $R$-branch wavelengths of gas-phase $\mathrm{CH}_{4}$ are indicated at the top, where the numbers refer to the lower rotational $J$ values. The gas-phase lines are further highlighted in Fig. 2. Additional solid-state absorption features are superposed on the $3.47 \mu \mathrm{m}$ band. The most secure identification is that of methanol (Allamandola et al. 1992). Other possible identifications are indicated by arrows, but these are not further discussed in this paper: ethanol (Allamandola et al. 1992), ethane (Boudin et al. 1998), formaldehyde (Schutte et al. 1996), and diffuse medium aliphatic C-H stretching modes (Sandford et al. 1991). The weak, broad inflection near $3.25 \mu \mathrm{m}$ is possibly due to absorption by aromatic hydrocarbons (Brooke et al. 1999).

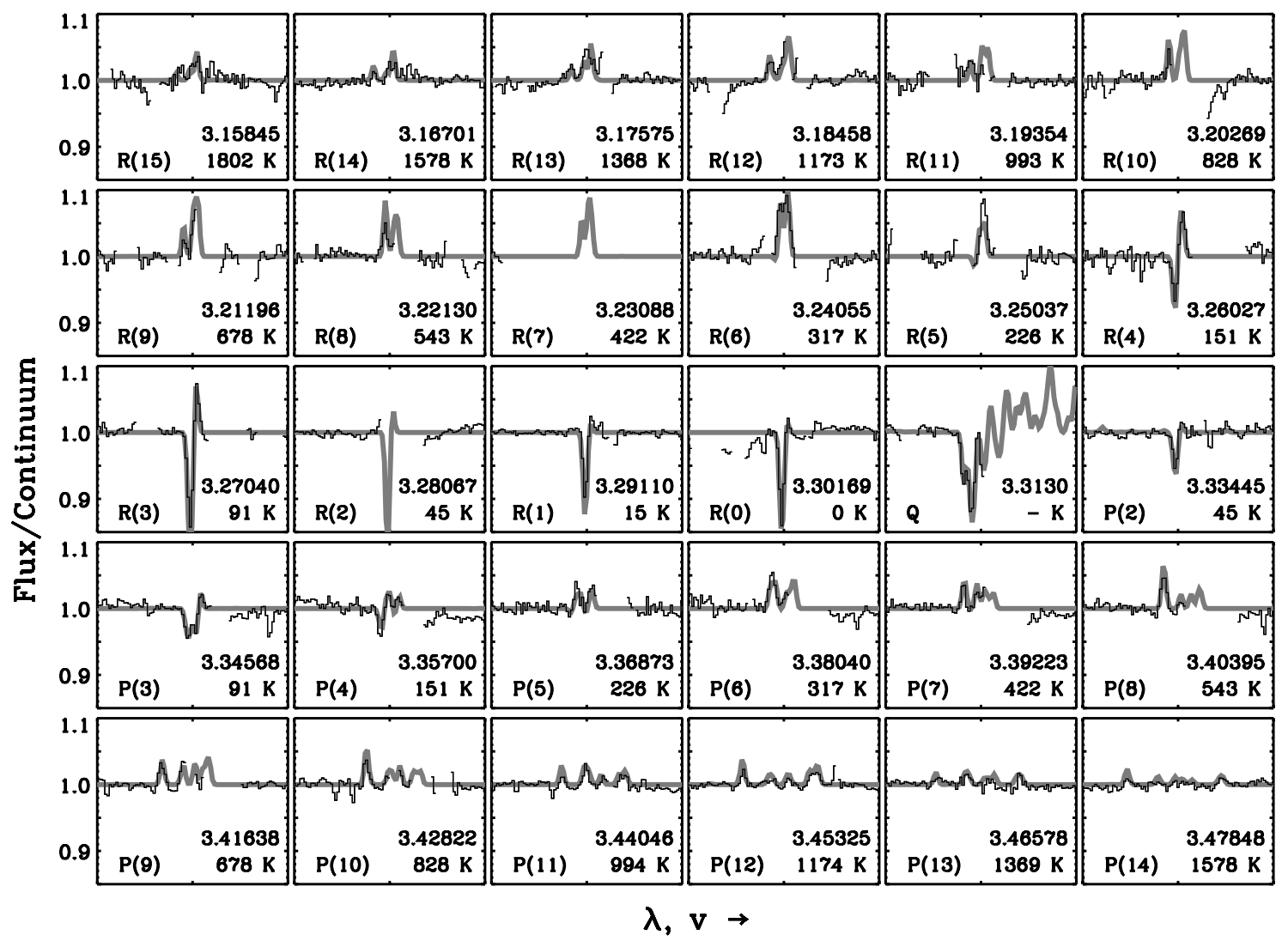

FIG. 2.-Magnification of the Keck/NIRSPEC spectrum of NGC 7538 IRS 9 in spectral regions surrounding gas-phase $\mathrm{CH}_{4}$ rovibrational lines $(\Delta \lambda=0.006 \mu \mathrm{m}$ or $\Delta v=530 \mathrm{~km} \mathrm{~s}^{-1}$ ). This is the same data as shown in Fig. 1, but on a flux/continuum scale. The gaps in the spectra are regions with less than $50 \%$ atmospheric transmission that were removed from the data. The thick gray line superposed on each observed spectrum depicts a two-component LTE model, composed of a $6 \mathrm{~km}$ $\mathrm{s}^{-1}$ blueshifted absorption component $\left(T=55 \mathrm{~K}, N=2.5 \times 10^{16} \mathrm{~cm}^{-2}\right)$ and an $8 \mathrm{~km} \mathrm{~s}^{-1}$ redshifted emission component $\left(T=600 \mathrm{~K}, N=5.8 \times 10^{16} \mathrm{~cm}^{-2} ;\right.$ Table 4$)$. The rovibrational transition, the lower rotational energy level (in $\mathrm{K}$ ), and the center wavelength (in $\mu \mathrm{m}$ ) are indicated in the bottom left and right corners of each panel, respectively. Note that $\mathrm{CH}_{4}$ emission is detected up to the highest excitation levels, i.e., up to $R(15)$ and $P(14)$. 
well. Finally, weak absorptions at 3.47 and $3.41 \mu \mathrm{m}$ are also close to the $\mathrm{C}-\mathrm{H}$ stretch mode of single-bonded carbon in $\mathrm{CH}$ and $\mathrm{CH}_{2}$ groups in aliphatic hydrocarbons, normally seen in diffuse lines of sight only (e.g., Pendleton et al. 1994).

\subsection{Gas-Phase Line Profiles at $R=25,000$ \\ 3.2.1. Gas-Phase $\mathrm{CH}_{4}$}

Rovibrational absorption and emission lines of gaseous $\mathrm{CH}_{4}$ are clearly detected toward NGC 7538 IRS 9 (Fig. 2). Although the telluric $\mathrm{CH}_{4}$ lines are deep, the large blueshift of NGC 7538 IRS 9 with respect to the Earth $(\S 2.1)$ separates many interstellar lines well. The lines appear mostly in absorption up to lower energy levels with $J=3$, and entirely in emission above $J=6$. Intermediate $J$ levels have P Cygni-like line profiles, i.e., redshifted emission and blueshifted absorption. Such line profiles are usually associated with expanding envelopes (see $\S 4.2$, however). Note that for $J>4$ multiple emission and absorption peaks appear for each $J$ level. These line splittings are intrinsic to the $\mathrm{CH}_{4}$ molecule and result from rotation-vibration interactions with the spin manifolds of the four equivalent $\mathrm{H}$ nuclei. Thus E-, F-, and A-type multiplets occur with spin statistical weights of $g_{s}=2,3$, and 5 , respectively.

The observed lines are analyzed using the 2001 update of the HITRAN 2000 database (Rothman et al. 2003; Brown et al. 2003). All HITRAN transitions contributing to each observed interstellar line are identified, and the number of transitions responsible for at least $90 \%$ of the line strength is indicated in column (3) of Tables 1 and 2. For example, at the resolution of our observations, the $P(11)$ transition is split into four separate emission lines, which are composed of some nine strong transitions. The contribution of each observed line to the total emission or absorption per $J$ level is indicated in column (4) of Tables 1 and 2 and is calculated in the optically thin limit as

$$
\frac{F_{J}^{\prime}}{F_{J}}=\frac{\sum_{i} g_{i J} A_{i J}}{\sum_{n} g_{n J} A_{n J}} \times 100 \%
$$

where $g_{i J}$ is the product of the rotation $(2 J+1)$ and nuclear spin $\left(g_{s}\right)$ statistical weights and $A_{i J}$ is the corresponding Einstein coefficient for transition $i$ at rotational level $J$. The summation over $i$ is for all transitions in the wavelength range of an observed emission line (col. [2] in Tables 1 and 2), while the summation over $n$ is for all transitions at rotational level $J$. For several $J$ levels only part of the emission or absorption is observed as some components lie in poor atmospheric regions. For example, only half of the $R(8)$ line flux is seen, and for the $Q$-branch the clearly detected absorption component represents only a few percent of the total $Q$-branch absorption. Finally, the observed emission-line fluxes $F_{J}^{\prime}$ listed in Table 1 are corrected for the underlying $\mathrm{H}_{2} \mathrm{O}$ absorption using a wide-coverage $I S O$ SWS spectrum (Whittet et al. 1996). The resulting continuum flux of $1.5 \mathrm{Jy}$ is corrected for foreground extinction using the Galactic extinction law (Draine 2003) and the $A_{V}$ derived in $\S 4.2$. Thus, the observed line fluxes are scaled to an unextincted continuum flux of $23.7 \mathrm{Jy}$.

In order to derive basic physical parameters, we construct a rotation diagram for the $\mathrm{CH}_{4}$ emission lines. The Boltzmann equation of a single transition $i$ at upper rotational level $J$ is

$$
\frac{N_{i J}}{g_{i J}}=\frac{N_{\text {tot }}}{Q\left(T_{\text {rot }}\right)} e^{-E_{i J} / k T_{\text {rot }}},
$$

TABLE 1

\begin{tabular}{|c|c|c|c|c|}
\hline $\begin{array}{c}\Delta v(J) \\
(1)\end{array}$ & $\begin{array}{c}\lambda^{\mathrm{a}} \\
(\mu \mathrm{m}) \\
(2)\end{array}$ & $\begin{array}{c}\text { Lines }^{\mathrm{b}} \\
\text { (3) }\end{array}$ & $\begin{array}{c}F_{J}^{\prime} / F_{J}^{\mathrm{c}} \\
(\%) \\
(4)\end{array}$ & $\begin{array}{c}F_{J}^{\prime \mathrm{d}} \\
\left(10^{-21} \mathrm{~W} \mathrm{~cm}^{-2}\right) \\
(5)\end{array}$ \\
\hline \multirow[t]{2}{*}{$R(15) \ldots \ldots \ldots \ldots \ldots \ldots$} & $3.15812-3.15864$ & 11 & 84 & $5.2(1.1)$ \\
\hline & $3.15767-3.15792$ & 3 & 13 & $<3.3$ \\
\hline \multirow[t]{2}{*}{$R(14) \ldots \ldots \ldots \ldots \ldots$} & $3.16677-3.16720$ & 13 & 73 & $3.8(0.7)$ \\
\hline & $3.16626-3.16665$ & 5 & 26 & $1.5(0.4)$ \\
\hline \multirow[t]{2}{*}{$R(13) \ldots \ldots \ldots \ldots \ldots \ldots$} & $3.17545-3.17595$ & 13 & 81 & $9.0(0.8)$ \\
\hline & $3.17498-3.17534$ & 4 & 16 & $4.3(0.8)$ \\
\hline \multirow[t]{2}{*}{$R(12) \ldots \ldots \ldots \ldots \ldots$} & $3.18435-3.18485$ & 14 & 74 & $13.6(3.0)$ \\
\hline & $3.18390-3.18428$ & 4 & 26 & $4.8(0.5)$ \\
\hline$R(11) \ldots \ldots \ldots \ldots \ldots$ & $3.19295-3.19325$ & 2 & 21 & $3.8(1.5)$ \\
\hline$R(10) \ldots \ldots \ldots \ldots \ldots \ldots$ & $3.20211-3.20253$ & 3 & 34 & $3.5(1.0)$ \\
\hline \multirow[t]{2}{*}{$R(9) \ldots \ldots \ldots \ldots \ldots$} & $3.21178-3.21229$ & 6 & 78 & $10.7(3.0)$ \\
\hline & $3.21146-3.21179$ & 2 & 22 & $3.0(0.8)$ \\
\hline$R(8) \ldots$ & $3.22087-3.22118$ & 3 & 47 & $4.8(0.8)$ \\
\hline$R(6) \ldots$ & $3.24014-3.24080$ & 6 & 100 & $25.1(1.5)$ \\
\hline$R(5) \ldots$. & $3.25019-3.25064$ & 4 & 100 & $12.5(1.4)$ \\
\hline$R(4) \ldots \ldots \ldots \ldots \ldots \ldots$ & $3.26017-3.26054$ & 4 & 100 & $7.5(1.0)$ \\
\hline$R(3) \ldots \ldots$ & $3.27035-3.27063$ & 3 & 100 & $6.6(0.5)$ \\
\hline$R(1) \ldots \ldots \ldots \ldots \ldots$ & $3.29105-3.29127$ & 1 & 100 & $1.5(0.5)$ \\
\hline$R(0) \ldots \ldots \ldots \ldots \ldots$ & $3.30165-3.30187$ & 1 & 100 & $1.5(0.6)$ \\
\hline$P(2) \ldots \ldots \ldots \ldots \ldots$ & $3.33435-3.33463$ & 2 & 100 & $1.8(0.4)$ \\
\hline$P(3) \ldots \ldots \ldots \ldots \ldots$ & $3.34573-3.34600$ & 3 & 45 & $2.1(0.5)$ \\
\hline \multirow[t]{2}{*}{$P(4) \ldots \ldots \ldots \ldots \ldots$} & $3.35708-3.35736$ & 1 & 23 & $1.9(0.6)$ \\
\hline & $3.35679-3.35703$ & 3 & 77 & $1.9(0.9)$ \\
\hline \multirow[t]{2}{*}{$P(5) \ldots \ldots \ldots \ldots \ldots \ldots$} & $3.36860-3.36901$ & 2 & 45 & $\geq 4.8$ \\
\hline & $3.36814-3.36860$ & 2 & 55 & $4.5(0.6)$ \\
\hline$P(6) \ldots \ldots \ldots \ldots \ldots$ & $3.37967-3.38022$ & 3 & 48 & $7.8(0.8)$ \\
\hline \multirow[t]{2}{*}{$P(7) \ldots \ldots \ldots \ldots \ldots \ldots$} & $3.39182-3.39230$ & 2 & 42 & $>5$ \\
\hline & $3.39138-3.39176$ & 2 & 32 & $3.5(0.4)$ \\
\hline \multirow[t]{2}{*}{$P(8) \ldots \ldots \ldots \ldots \ldots \ldots$} & $3.40379-3.40411$ & 1 & 14 & $\geq 1.6$ \\
\hline & $3.40318-3.40370$ & 3 & 48 & $6.8(0.4)$ \\
\hline \multirow[t]{2}{*}{$P(9) \ldots \ldots \ldots \ldots \ldots \ldots$} & $3.41577-3.41613$ & 2 & 18 & $>2.7$ \\
\hline & $3.41509-3.41560$ & 2 & 22 & $4.1(0.4)$ \\
\hline$P(10) \ldots \ldots \ldots \ldots \ldots$ & $3.42719-3.42772$ & 3 & 35 & $4.2(0.7)$ \\
\hline \multirow[t]{4}{*}{$P(11) \ldots \ldots \ldots \ldots \ldots$} & $3.44128-3.44172$ & 3 & 30 & $3.2(0.4)$ \\
\hline & $3.44072-3.44109$ & 1 & 11 & $1.6(0.2)$ \\
\hline & $3.44009-3.44057$ & 3 & 37 & $3.9(0.2)$ \\
\hline & $3.43944-3.43982$ & 2 & 22 & $2.0(0.2)$ \\
\hline \multirow[t]{4}{*}{$P(12) \ldots \ldots \ldots \ldots \ldots$} & $3.45380-3.45441$ & 4 & 43 & $4.7(0.3)$ \\
\hline & $3.45313-3.45347$ & 2 & 13 & $1.4(0.3)$ \\
\hline & $3.45252-3.45296$ & 2 & 16 & $1.1(0.4)$ \\
\hline & $3.45175-3.45208$ & 3 & 27 & $1.9(0.3)$ \\
\hline \multirow{4}{*}{$P(13) \ldots \ldots \ldots \ldots \ldots$} & $3.46664-3.46711$ & 3 & 23 & $1.1(0.4)$ \\
\hline & $3.46566-3.46629$ & 3 & 31 & $0.8(0.3)$ \\
\hline & $3.46501-3.46548$ & 3 & 28 & $1.8(0.3)$ \\
\hline & $3.46406-3.46457$ & 2 & 17 & $0.8(0.3)$ \\
\hline \multirow[t]{4}{*}{$P(14) \ldots \ldots \ldots \ldots \ldots \ldots$} & $3.47952-3.48004$ & 3 & 22 & $1.9(0.4)$ \\
\hline & $3.47814-3.47899$ & 4 & 35 & $0.6(0.3)$ \\
\hline & $3.47757-3.47796$ & 2 & 16 & $<0.8$ \\
\hline & $3.47659-3.47707$ & 3 & 27 & $1.7(0.4)$ \\
\hline
\end{tabular}

$\mathrm{CH}_{4}$ Emission-Line Strengths toward NGC 7538 IRS 9

${ }^{a}$ Integration region after correction for $8 \mathrm{~km} \mathrm{~s}^{-1}$ redshift.

b Number of blended lines in integration interval.

c Percentage of emission with respect to total emission for this upper $J$ level.

d $1 \sigma$ errors in parentheses.

where $N_{i J}$ and $E_{i J}$ are the column density and the energy of the upper level of a single multiplet component, respectively. Assuming that collisional excitation is applicable, $E_{i J}$ includes a vibrational $v=1$ excitation energy of $4348 \mathrm{~K}$ (Lide 1994); $N_{\text {tot }}$ and $T_{\text {rot }}$ are the total $\mathrm{CH}_{4}$ column density and rotational temperature. Throughout this paper we take the rovibrational partition function, $Q\left(T_{\text {rot }}\right)$, from the HITRAN database, which 
TABLE 2

$\mathrm{CH}_{4}$ Absorption-Line Strengths toward NGC 7538 IRS 9

\begin{tabular}{|c|c|c|c|c|}
\hline $\begin{array}{c}\Delta v(J) \\
(1)\end{array}$ & $\begin{array}{c}\lambda^{\mathrm{a}} \\
(\mu \mathrm{m}) \\
(2)\end{array}$ & $\begin{array}{c}\text { Lines }^{b} \\
\text { (3) }\end{array}$ & $\begin{array}{c}F_{J}^{\prime} / F_{J}^{\mathrm{c}} \\
(\%) \\
(4)\end{array}$ & $\begin{array}{c}\text { Equivalent Width } \\
\left(10^{-3} \mathrm{~cm}^{-1}\right) \\
(5)\end{array}$ \\
\hline$R(4) \ldots \ldots \ldots \ldots \ldots$ & $3.26003-3.26031$ & 3 & 77 & $10.0(1.4)$ \\
\hline$R(3) \ldots \ldots \ldots \ldots$ & $3.27019-3.27051$ & 3 & 100 & $12(1)$ \\
\hline$R(1) \ldots \ldots \ldots \ldots$ & $3.29092-3.29121$ & 1 & 100 & $12.7(0.4)$ \\
\hline$R(0) \ldots \ldots \ldots \ldots$ & $3.30151-3.30179$ & 1 & 100 & $17.6(1.0)$ \\
\hline$Q \ldots \ldots \ldots \ldots \ldots \ldots$ & $3.31234-3.31296$ & 4 & 2 & $35(6)$ \\
\hline$P(2) \ldots \ldots \ldots \ldots$ & $3.33423-3.33453$ & 2 & 100 & $6.5(0.8)$ \\
\hline$P(3) \ldots \ldots \ldots \ldots$ & $3.34530-3.34584$ & 3 & 100 & $12.3(0.7)$ \\
\hline$P(4) \ldots \ldots \ldots \ldots \ldots$ & $3.35660-3.35694$ & 3 & 61 & $5.9(0.8)$ \\
\hline
\end{tabular}

${ }^{\text {a }}$ Integration region after correction for $6 \mathrm{~km} \mathrm{~s}^{-1}$ blueshift.

b Number of blended lines in integration interval.

${ }^{\mathrm{c}}$ Percentage of absorption with respect to total absorption for this lower $J$ level.

d $1 \sigma$ errors in parenthesess.

is applicable at $T_{\text {rot }}=70-3000 \mathrm{~K}$ (Fischer et al. 2003). At lower temperatures we calculate $Q\left(T_{\text {rot }}\right)$ from lines in the HITRAN database. In the optically thin limit, the flux $F_{J}^{\prime}$ of a partial $J$ transition is obtained by summation over all multiplet lines,

$$
F_{J}^{\prime}=\sum_{i} F_{i J}
$$

and thus the observed $F_{J}^{\prime}$ relates to equation (2) as

$$
\frac{4 \pi F_{J}^{\prime}}{h c \nu_{J} \Omega \sum_{i} g_{i J} A_{i J}}=\frac{N_{\text {tot }}}{Q\left(T_{\text {rot }}\right)} e^{-E_{J} / k T_{\text {rot }}} .
$$

Here we make use of the fact that the upper energy level $E_{J}$ and transition frequency $\nu_{J}$ are nearly the same for all multiplet lines (to within $0.1 \%$ ); $\Omega$ is the size of the emitting region, for which we assume the value of $4.3 \times 10^{-14}$ sr derived from $\mathrm{CO}$ emission lines, 2 orders of magnitude smaller than the solid angle corresponding to the 0.42 wide slit $(\S 4.2)$. The rotation diagram constructed from the observed $\mathrm{CH}_{4}$ emission lines indicates a rotational temperature of $600 \pm 100 \mathrm{~K}$ (Fig. 3). Although $T_{\text {rot }}$ is well constrained, the derived column density of $5.8_{-3}^{+16} \times 10^{16} \mathrm{~cm}^{-2}$ is accurate to within a factor of $\sim 3$ only $(3 \sigma$ errors).

To further illustrate the goodness offit, to investigate possible optical depth effects, and to model the complex blend of $\mathrm{CH}_{4}$ absorption and emission lines, we have also constructed singletemperature LTE models. The level populations are calculated according to equation (2). Emission-line fluxes and absorption equivalent widths at any optical depth are calculated following Genzel (1992) and Spitzer (1978), respectively, applying the Voigt function for the line profiles. The observed line profiles are fitted by adding a single-temperature redshifted emission model to a single-temperature, blueshifted absorption model. We find that a blueshift of $6 \mathrm{~km} \mathrm{~s}^{-1}$ and a redshift of $8 \mathrm{~km} \mathrm{~s}^{-1}$ are needed, and that both components are unresolved at the spectral resolution of $12 \mathrm{~km} \mathrm{~s}^{-1}$. The validity of the temperature and column density derived from the emission-line rotation diagram is confirmed by the relatively low model line optical depth; for example, at a Doppler parameter of $b_{\mathrm{D}}=1 \mathrm{~km} \mathrm{~s}^{-1}, N_{\text {tot }}=$ $5.8 \times 10^{16} \mathrm{~cm}^{-2}$, and $T=600 \mathrm{~K}$, the peak optical depth is 0.33 [in the $R(8)$ line]. The absorption column is well constrained to $N\left(\mathrm{CH}_{4}\right)=3 \pm 1 \times 10^{16} \mathrm{~cm}^{-2}$ at $T_{\mathrm{ex}}=55 \pm 15 \mathrm{~K}$ for intrinsic

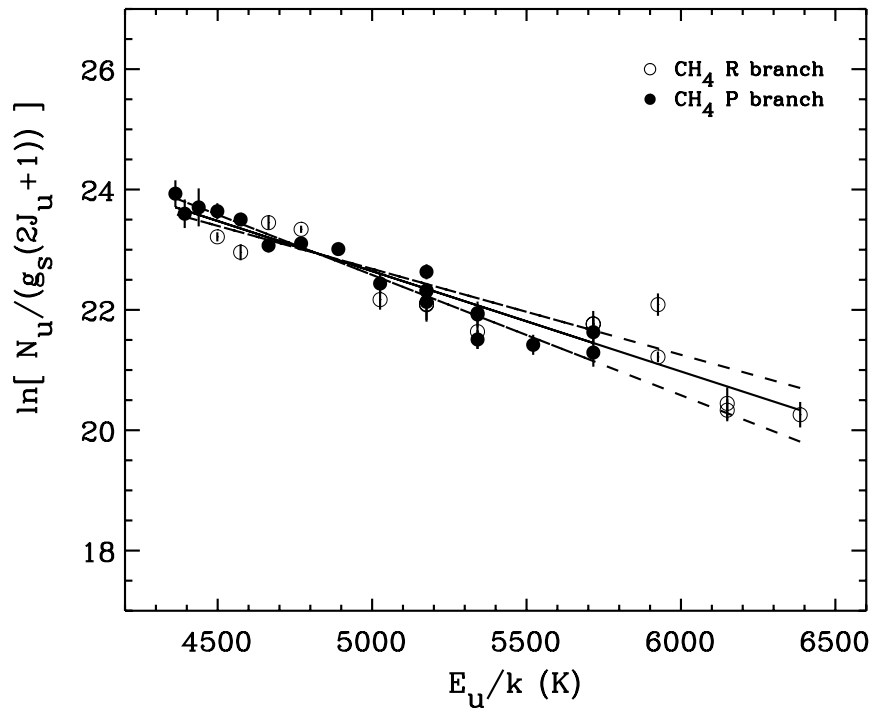

FIG. 3.-Rotation diagram of $\mathrm{CH}_{4}$ emission lines toward NGC 7538 IRS 9. $P$-branch lines are shown by filled circles, and $R$-branch lines are shown by open circles. The solid line represents a least-squares best fit to the data corresponding to a rotational temperature of $600 \mathrm{~K}$ at a column density of $5.8 \times$ $10^{16} \mathrm{~cm}^{-2}$ in a solid angle of $4.3 \times 10^{-14} \mathrm{sr}$. The dashed lines represent extreme $(3 \sigma)$ physical parameters that still fit the data: $T_{\text {rot }}=500 \mathrm{~K}$ and $N\left(\mathrm{CH}_{4}\right)=$ $20 \times 10^{16} \mathrm{~cm}^{-2}$, and $T_{\text {rot }}=700 \mathrm{~K}$ and $N\left(\mathrm{CH}_{4}\right)=2.6 \times 10^{16} \mathrm{~cm}^{-2}$.

line widths of $b_{\mathrm{D}}=0.5-3 \mathrm{~km} \mathrm{~s}^{-1}$ (Table 4). The narrowest lines are optically thick $\left(\tau=5\right.$ at $\left.b_{\mathrm{D}}=0.5 \mathrm{~km} \mathrm{~s}^{-1}\right)$, while they are optically thin for $b_{\mathrm{D}}>1 \mathrm{~km} \mathrm{~s}^{-1}$. We take $b_{\mathrm{D}}=3 \mathrm{~km} \mathrm{~s}^{-1}$ as a preferred value throughout this paper, because the larger line widths are usually preferred toward NGC 7538 IRS 9 (Mitchell et al. 1990; Boonman \& van Dishoeck 2003), but values of $b_{\mathrm{D}}>$ $3 \mathrm{~km} \mathrm{~s}^{-1}$ are inconsistent with the unresolved $\mathrm{CH}_{4}$ lines observed. The physical parameters derived from the $\mathrm{CH}_{4}$ stretching mode are in good agreement with the parameters from the bending mode at $7.67 \mu \mathrm{m}$ in lower resolution spectra (Lacy et al. 1991; Boogert et al. 1998).

Finally, absorption or emission lines of the ${ }^{13} \mathrm{CH}_{4}$ and $\mathrm{CH}_{3} \mathrm{D}$ isotopes have not been detected. Study of the ${ }^{13} \mathrm{CH}_{4}$ lines is complicated, because the isotope wavelength shift is comparable to the separation of $J$ levels, and thus the ${ }^{13} \mathrm{CH}_{4}$ and ${ }^{12} \mathrm{CH}_{4}$ lines are blended. The column density upper limits are a factor of 37 smaller compared to the $\mathrm{CH}_{4}$ column density. At ${ }^{12} \mathrm{C} /{ }^{13} \mathrm{C}$ and $\mathrm{H} / \mathrm{D}$ isotope ratios of at least 50 in dense clouds (Wilson \& Rood 1994; Ceccarelli et al. 2001), these upper limits are not significant.

\subsubsection{Gas-Phase CO}

At first glance, the line profiles of the fundamental rovibrational $\mathrm{CO}$ transitions at $4.7 \mu \mathrm{m}$, as published in Mitchell et al. (1990) and Boogert et al. (2002), look similar to those of $\mathrm{CH}_{4}$. They show $\mathrm{P}$ Cygni profiles as well, in ${ }^{12} \mathrm{CO},{ }^{13} \mathrm{CO}$, and perhaps even the $\mathrm{C}^{18} \mathrm{O}$ isotope, with the strength of emission versus absorption increasing for higher $J$ levels. On closer inspection, however, the absorption profiles of $\mathrm{CO}$ and $\mathrm{CH}_{4}$ differ. The low- $J$ isotopic $C O$ lines peak at $0 \mathrm{~km} \mathrm{~s}^{-1}$, compared to $-6 \mathrm{~km} \mathrm{~s}^{-1}$ for $\mathrm{CH}_{4}$ (Fig. $\left.4 a\right)$. Higher $J \mathrm{CO}$ lines $\left(E_{\text {low }} \geq 100 \mathrm{~K}\right)$, on the other hand, are dominated by absorption at blueshifted velocities $\geq 16 \mathrm{~km} \mathrm{~s}^{-1}$, which is absent in $\mathrm{CH}_{4}$ (Fig. $4 b$ ). This provides clues to the formation and evolution history of $\mathrm{CH}_{4}$ (§ 4.1), and in this context it is relevant to quantify the physical conditions of the absorbing and emitting $\mathrm{CO}$ gas as well ( Table 4). 


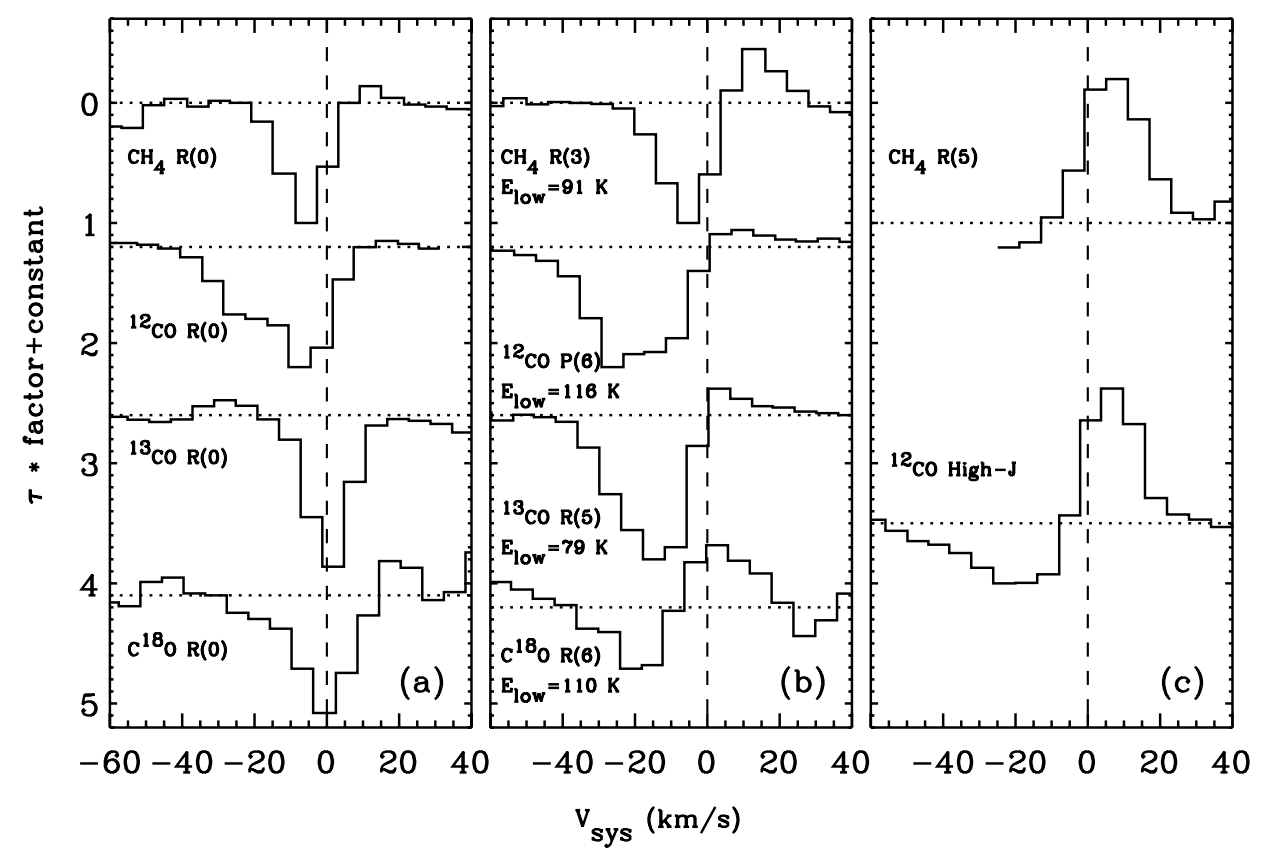

FIG. 4.-Line profiles of $\mathrm{CH}_{4}$ and $\mathrm{CO}$ isotopologues on a velocity scale corrected for the earth and heliocentric velocity. Lines of similar lower energy level are plotted: $(a) 0 \mathrm{~K}$ and $(b) \sim 100 \mathrm{~K}$. For reference, we have indicated the systemic velocity with a vertical dashed line. Note the very different profile of $\mathrm{CH}_{4}$ compared to $\mathrm{CO}$ lines. The $-16 \mathrm{~km} \mathrm{~s}^{-1}$ outflow is absent in $\mathrm{CH}_{4}$, and the $R(0) \mathrm{CH}_{4}$ line does not peak at the systemic velocity, indicating that $\mathrm{CO}$ and $\mathrm{CH}_{4}$ have a different chemical origin and evolution ( $\S 4.1)$. (c) Emission-dominated $\mathrm{CH}_{4}$ and $\mathrm{CO}$ line profiles. The high- $J \mathrm{CO}$ line profile toward NGC 7538 IRS 9 is an average over the individual lines $P(27), P(28), P(30)$, and $P(34)$. The emission lines clearly peak at $+8 \mathrm{~km} \mathrm{~s}^{-1}$, opposite what is expected for P Cygni line profiles ( $\left.\S 4.2\right)$.

We analyzed the $R=25,000 \mathrm{Keck} / \mathrm{NIRSPEC}$ spectrum of Boogert et al. (2002) and arrive at different conclusions compared to Mitchell et al. $(1990,1991)$. First, and most importantly, in the Keck/NIRSPEC data blueshifted absorption at $16 \mathrm{~km} \mathrm{~s}^{-1}$ is seen up to $J$ levels as high as 20 , but only up to $J=3$ in Mitchell et al. (1991). In the rotation diagram constructed from the integrated ${ }^{13} \mathrm{CO}$ absorption equivalent widths, this high-velocity gas is responsible for the linear part above energies of $100 \mathrm{~K}$ (Fig. 5; Table 3). From this a column density of $N\left({ }^{12} \mathrm{CO}\right)=$ $3.5 \pm 1.0 \times 10^{18} \mathrm{~cm}^{-2}$ at a temperature of $T_{\text {rot }}=270 \pm 100 \mathrm{~K}$ $(3 \sigma)$ is derived. The column density is comparable to that found by Mitchell et al. (1991), but $T_{\text {rot }}$ is a factor of 10 higher in the Keck/NIRSPEC data. A second difference is that $\mathrm{C}^{18} \mathrm{O}$ lines are detected in the high S/N Keck/NIRSPEC data. The ${ }^{13} \mathrm{CO} / \mathrm{C}^{18} \mathrm{O}$ absorption-line ratios of corresponding $J$ levels are equal to the interstellar isotope ratio of 6.9 at large Galactocentric radii (Wilson \& Rood 1994) within a $20 \%$ error margin (see the $\mathrm{C}^{18} \mathrm{O}$ data points in the ${ }^{13} \mathrm{CO}$ rotation diagram; Fig. 5). An important consequence is that reliable $\mathrm{CO}$ column densities can be derived, and thus reliable $\mathrm{CH}_{4}$ abundances in the various velocity ranges (§ 4.1). The steep part of the ${ }^{13} \mathrm{CO}$ rotation diagram, corresponding to gas at velocities of -8 to $+11 \mathrm{~km} \mathrm{~s}^{-1}$, indicates $T_{\text {rot }}=20 \pm$ $7 \mathrm{~K}$ and a column density of $N\left({ }^{12} \mathrm{CO}\right)=3.5 \pm 1.0 \times 10^{18} \mathrm{~cm}^{-2}$, which is a factor of 4 less compared to that found by Mitchell et al. (1990). This is due to our factor of 2 smaller measured equivalent widths and presumably lower assumed line opacity.

For the emission lines, the observed ${ }^{12} \mathrm{CO} /{ }^{13} \mathrm{CO}$ peak strength ratio is $6 \pm 2$ at intermediate upper $J$ levels of $7-15$. Using the standard radiative transfer equation, the peak optical depth of the ${ }^{12} \mathrm{CO}$ emission lines, $\tau_{12}$ can be derived from

$$
\frac{F\left({ }^{12} \mathrm{CO}\right)}{F\left({ }^{13} \mathrm{CO}\right)}=\frac{1-e^{-\tau_{12}}}{1-e^{-\tau_{12} / 80}} .
$$

At a ${ }^{12} \mathrm{CO} /{ }^{13} \mathrm{CO}$ isotope ratio of 80 (Boogert et al. 2002), it follows that $\tau_{12}=13 \pm 4$. Such an optical depth corresponds to a column density of $N\left({ }^{12} \mathrm{CO}\right)=3.2 \pm 1.0 \times 10^{18} \mathrm{~cm}^{-2}$ at an intrinsic line width of $b_{\mathrm{D}}=3 \mathrm{~km} \mathrm{~s}^{-1}$. We further discuss the origin of the emitting gas in $\S 4.2$.

\subsection{The Solid $\mathrm{CH}_{4}$ Absorption Bands}

Solid $\mathrm{CH}_{4}$ has been detected by its $\mathrm{C}-\mathrm{H}$ bending mode at $7.67 \mu \mathrm{m}$ in ISO SWS observations of NGC 7538 IRS 9

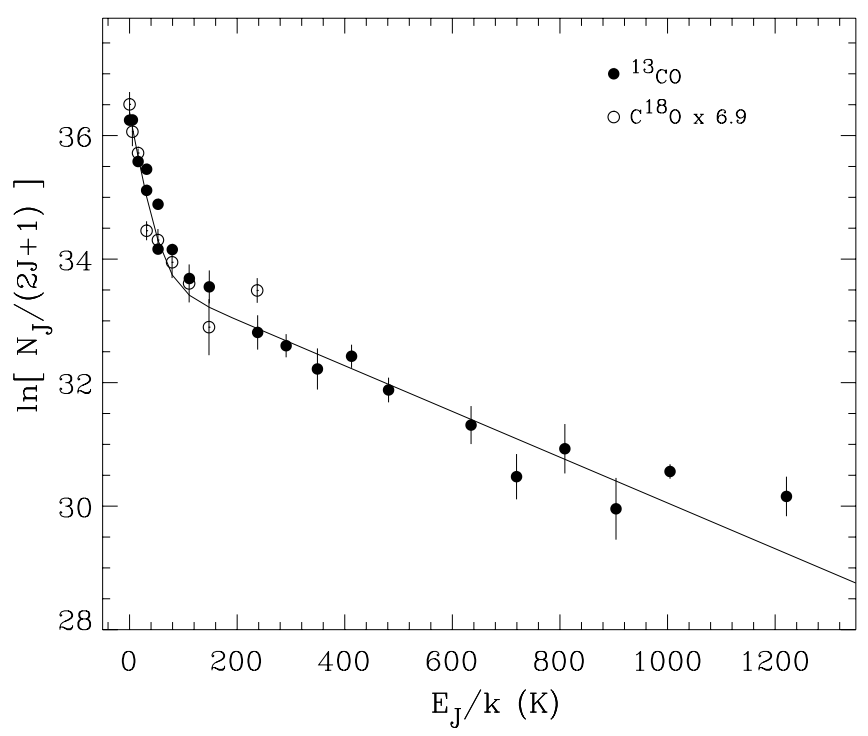

FIG. 5.- Rotation diagram of ${ }^{13} \mathrm{CO}$ ( filled circles) and $\mathrm{C}^{18} \mathrm{O}$ (open circles) absorption lines toward NGC 7538 IRS 9. The $\mathrm{C}^{18} \mathrm{O}$ column densities have been scaled up by a factor of 6.9 to match the abundance of interstellar ${ }^{13} \mathrm{CO}$ (Wilson \& Rood 1994). The good agreement of these isotopes in this plot implies that the ${ }^{13} \mathrm{CO}$ absorption lines are optically thin. The solid line represents a two-component gas with rotational temperatures of 20 and $270 \mathrm{~K}$ both at a ${ }^{13} \mathrm{CO}$ column density of $4.65 \times 10^{16} \mathrm{~cm}^{-2}$. Note that the $20 \mathrm{~K}$ gas absorbs at the systemic velocity, while the warm component absorbs at much higher velocity (Fig. 4). 
TABLE 3

Gas-Phase CO Absorption toward NGC 7538 IRS 9

\begin{tabular}{|c|c|c|}
\hline \multirow[b]{2}{*}{$\Delta v(J)^{\mathrm{a}}$} & \multicolumn{2}{|c|}{$\begin{array}{c}\text { EQUIVALENT WIDTH } \\
\quad\left(10^{-3} \mathrm{~cm}^{-1}\right)\end{array}$} \\
\hline & ${ }^{13} \mathrm{CO}$ & $\mathrm{C}^{18} \mathrm{O}$ \\
\hline$R(18) \ldots \ldots$ & $2(1)$ & $\ldots$ \\
\hline$R(17) \ldots \ldots$ & $5(2)$ & $\cdots$ \\
\hline$R(16) \ldots \ldots$ & $3(1.1)$ & $\ldots$ \\
\hline$R(15) \ldots \ldots$ & $6.5(2)$ & $\ldots$ \\
\hline$R(13) \ldots \ldots \ldots$ & $10(2)$ & $\ldots$ \\
\hline$R(12) \ldots \ldots \ldots$ & $16(3)$ & $\ldots$ \\
\hline$R(11) \ldots \ldots$ & $12(4)$ & $<4.5$ \\
\hline$R(10) \ldots \ldots$ & $16(3)$ & $<4.5$ \\
\hline$R(9) \ldots \ldots$ & $18(5)$ & $5(1)$ \\
\hline$R(7) \ldots \ldots \ldots$ & $30(8)$ & $2.2(1.0)$ \\
\hline$R(6) \ldots \ldots$ & $30(2)$ & $3.9(1.2)$ \\
\hline$R(5) \ldots \ldots \ldots \ldots \ldots$ & $41(3)$ & $4.7(1.2)$ \\
\hline$R(4) \ldots \ldots$ & $71(4)$ & $5.6(1.0)$ \\
\hline$R(3) \ldots \ldots$ & $71(4)$ & $5.2(0.8)$ \\
\hline$R(2) \ldots \ldots \ldots$ & $\ldots$ & $13.7(1)$ \\
\hline$R(0) \ldots \ldots$ & $55(3)$ & $10(2)$ \\
\hline$P(1) \ldots \ldots$ & $55(2)$ & $6.4(1.5)$ \\
\hline$P(2) \ldots \ldots$ & $56(3)$ & $\ldots$ \\
\hline$P(3) \ldots \ldots$ & $74(2)$ & $\ldots$ \\
\hline 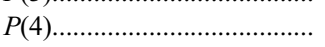 & $27(2)$ & $\ldots$ \\
\hline$P(18) \ldots \ldots$ & $<15$ & $\ldots$ \\
\hline$P(19) \ldots \ldots$ & $3.4(0.4)$ & $\ldots$ \\
\hline$P(21)$ & $2.5(0.8)$ & $\ldots$ \\
\hline$P(22) \ldots \ldots \ldots \ldots$. & $<6$ & $\ldots$ \\
\hline
\end{tabular}

a Transitions not listed have not been observed, are in a poor atmospheric region, or are blended with a brighter isotope.

${ }_{1} \sigma$ errors in parentheses.
(Boogert et al. 1996). The peak position and width of the interstellar band are well fitted with an amorphous, low-temperature $(10 \mathrm{~K})$ laboratory ice in which $\mathrm{CH}_{4}$ is diluted in an $\mathrm{H}_{2} \mathrm{O}$ matrix. This same laboratory ice gives an excellent fit to the new $3.32 \mu \mathrm{m}$ absorption feature detected in the Keck/NIRSPEC and ISO SWS spectra, not only in peak position and width, but also to the peak optical depth of 0.1 . We thus attribute this interstellar band in its entirety to the $\mathrm{C}-\mathrm{H}$ stretching mode of solid $\mathrm{CH}_{4}$ in an $\mathrm{H}_{2} \mathrm{O}$-rich ice (Fig. 6). A mismatch with the observed ISO SWS spectrum on the low-wavelength wing is attributed to emission by the strong $Q$-branch of gaseous $\mathrm{CH}_{4}$ (Fig. 6). Indeed, a better match is obtained after subtracting the model constructed from the observed $P$ - and $R$-branch lines from the ISO SWS spectrum (§3.2.1). In contrast, contamination of gasphase emission to the $7.67 \mu \mathrm{m}$ ice band is small because the continuum emission at this wavelength is more than an order of magnitude brighter compared to the continuum at $3.32 \mu \mathrm{m}$ (Whittet et al. 1996). Indeed, a weak inflection at $7.66 \mu \mathrm{m}$ is due to $Q$-branch absorption. Simultaneous fits of the $\mathrm{C}-\mathrm{H}$ stretch and bend modes highlight the good fit of $\mathrm{CH}_{4}: \mathrm{H}_{2} \mathrm{O}$ ices (Fig. 6). Pure $\mathrm{CH}_{4}$ ices and mixtures with $\mathrm{CO}$, otherwise highly abundant in this line of sight (Tielens et al. 1991; Boogert et al. 2002), can be excluded at a high confidence level. Note that the $\mathrm{C}-\mathrm{H}$ stretching mode of $\mathrm{CH}_{4}$ better discriminates between polar and apolar ices than does the bending mode. Mixtures with other high-dipole molecules, such as $\mathrm{NH}_{3}$ and $\mathrm{CH}_{3} \mathrm{OH}$, do not provide equally good fits compared to $\mathrm{H}_{2} \mathrm{O}$, but at present we cannot exclude combinations of these polar species.

\section{DISCUSSION}

\subsection{Astrochemical Implications}

The detections of both the $3.32 \mu \mathrm{m}$ stretch and the $7.67 \mu \mathrm{m}$ bend modes firmly establish the presence of both gaseous and solid $\mathrm{CH}_{4}$ in the interstellar medium. The gas-state+solid-state abundance averaged along the line of sight of the massive

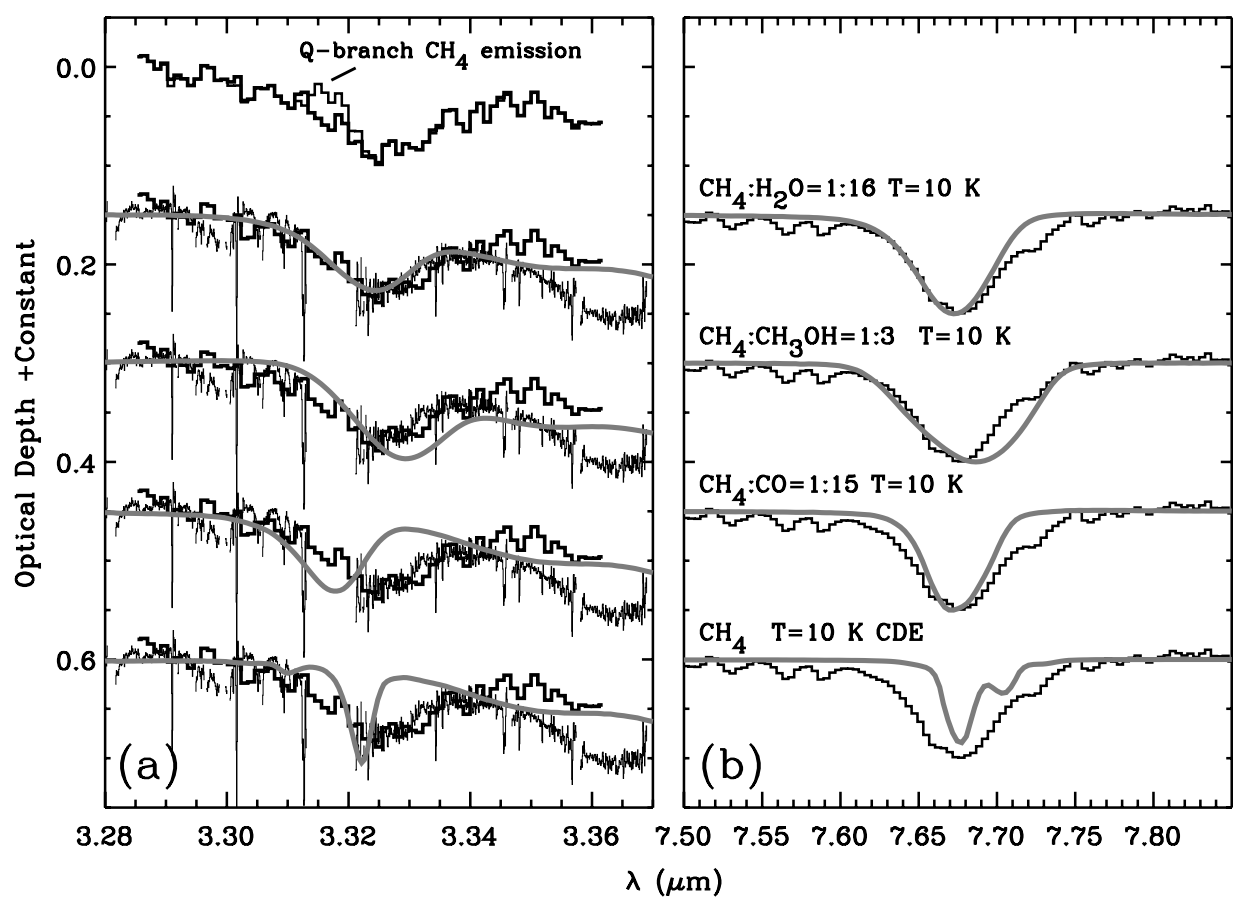

FIG. 6. - (a) Stretching mode of solid $\mathrm{CH}_{4}$ observed with ISO SWS (thick histogram) and KECK/NIRSPEC (thin histogram), compared to a number of laboratory ice simulations (smooth gray line). At the very top the effect of removing the $Q$-branch emission of gas-phase $\mathrm{CH}_{4}$ is shown. $(b)$ The $\mathrm{CH}_{4}$ bending mode is fitted with the same laboratory ice, without scaling the peak optical depth. Best fits are obtained with $\mathrm{H}_{2} \mathrm{O}$-rich ices. Note the poor fit of $\mathrm{CO}_{\text {-rich }}$ ices to the $\mathrm{CH}_{4}$ stretching mode. 
TABLE 4

$\mathrm{CH}_{4}$ and CO Physical Parameters toward NGC 7538 IRS 9

\begin{tabular}{|c|c|c|c|c|c|c|c|}
\hline Species & Location $^{a}$ & Phase $^{\mathrm{b}}$ & $\begin{array}{c}V^{\mathrm{c}} \\
\left(\mathrm{km} \mathrm{s}^{-1}\right)\end{array}$ & $\begin{array}{l}T_{\mathrm{ex}}^{\mathrm{d}} \\
(\mathrm{K})\end{array}$ & $\begin{array}{c}N^{\mathrm{d}} \\
\left(10^{17} \mathrm{~cm}^{-2}\right)\end{array}$ & $\begin{array}{c}N / N_{\mathrm{H}}^{\mathrm{d}, \mathrm{e}} \\
\left(10^{-6}\right)\end{array}$ & Notes \\
\hline $\mathrm{CH}_{4} \ldots \ldots \ldots \ldots \ldots \ldots$ & Outflow & Gas/absorption & -16 & 270 & $<0.1$ & $<0.4$ & Assumed $T$ \\
\hline $\mathrm{CH}_{4} \ldots \ldots \ldots \ldots \ldots \ldots \ldots \ldots$ & Core & Gas/absorption & -6 & $55 \pm 15$ & $0.3 \pm 0.1$ & $\geq 3$ & $b_{\mathrm{D}}=0.5-3 \mathrm{~km} \mathrm{~s}^{-1}$ \\
\hline $\mathrm{CH}_{4} \ldots \ldots \ldots \ldots \ldots \ldots \ldots$ & Envelope & Gas/absorption & 0 & 20 & $<0.1$ & $<0.3$ & Assumed $T$ \\
\hline $\mathrm{CH}_{4} \ldots \ldots \ldots \ldots \ldots \ldots$ & Core & Gas/emission & +8 & $600 \pm 100$ & $0.58_{-0.3}^{+1.6}$ & $1.9_{-0.9}^{+7.2}$ & $4.3 \times 10^{-14} \mathrm{sr}^{\mathrm{f}}$ \\
\hline $\mathrm{CH}_{4}{ }^{\mathrm{g}} \ldots \ldots \ldots \ldots \ldots \ldots$ & Envelope & Ice/absorption & $\ldots$ & $\ldots$ & $1.3 \pm 0.1$ & $2.1 \pm 0.7^{\mathrm{h}}$ & \\
\hline $\mathrm{CO}$ & Outflow & Gas/absorption & -16 & $270 \pm 100$ & $35 \pm 10$ & 100 & Optically thin ${ }^{13} \mathrm{CO}$ \\
\hline $\mathrm{CO}$ & Core & Gas/absorption & -6 & 55 & $<10$ & 100 & Assumed $T$ \\
\hline $\mathrm{CO}$ & Envelope & Gas/absorption & 0 & $20 \pm 7$ & $35 \pm 10$ & 100 & Optically thin ${ }^{13} \mathrm{CO}$ \\
\hline $\mathrm{CO}$ & Core & Gas/emission & +8 & $500 \pm 150$ & $30 \pm 10^{\mathrm{i}}$ & 100 & $4.3 \times 10^{-14} \mathrm{sr}^{\mathrm{f}}$ \\
\hline $\mathrm{CO}^{\mathrm{j}} \ldots \ldots \ldots \ldots \ldots \ldots \ldots$ & Envelope & Ice/absorption & $\ldots$ & $\ldots$ & $18 \pm 2$ & $36 \pm 10^{\mathrm{h}}$ & \\
\hline
\end{tabular}

\footnotetext{
a Assumed location from velocity; see also $\S \S 4.1$ and 4.2.

${ }^{b}$ In gas or ices, in emission or absorption.

c Velocity with respect to systemic $V_{\mathrm{lsr}}=57 \mathrm{~km} \mathrm{~s}^{-1}$.

${ }^{\mathrm{d}}$ Errors are $3 \sigma$.

e Assuming $N_{\mathrm{H}} / \mathrm{CO}=1 \times 10^{4}$ from Lacy et al. (1994).

$\mathrm{f}$ Solid angle derived from $\mathrm{CO}$ emission.

${ }^{\mathrm{g}}$ Boogert et al. (1998).

${ }^{\mathrm{h}}$ Ice abundance with respect to $\mathrm{CO}$ gas+ice abundance in envelope.

${ }^{i}$ Uncertainty in column dominated by uncertainty in line opacity.

j Boogert et al. (2002).
}

protostar NGC 7538 IRS 9 is $X\left[\mathrm{CH}_{4}\right]=2 \times 10^{-6}$, and the absorption gas-state/solid-state abundance ratio is 0.23 . A combined high-resolution $\mathrm{CO} / \mathrm{CH}_{4}$ line profile analysis shows that the abundance, as well as the gas-state/solid-state abundance ratio of $\mathrm{CH}_{4}$, varies locally by an order of magnitude, however (Table 4). An ice+gas abundance of a few $\times 10^{-6}$ is found in the outer envelope (systemic velocity), the inner envelope $\left(-6 \mathrm{~km} \mathrm{~s}^{-1}\right)$, and the compact emission component $(+8 \mathrm{~km}$ $\mathrm{s}^{-1}$ ). In the outer envelope, at $20 \mathrm{~K}$, the coldest component along the line of sight, at least $90 \%$ of the $\mathrm{CH}_{4}$ is frozen on grains. Within the envelope a segregation is present as well; $\mathrm{CH}_{4}$ is intimately mixed with $\mathrm{H}_{2} \mathrm{O}$ and $\mathrm{CH}_{3} \mathrm{OH}$ rather than with $\mathrm{CO}$. In the warm, low-velocity inner regions of NGC 7538 IRS $9, \mathrm{CH}_{4}$ is mostly present in the gas phase. These enhanced abundances strongly contrast with the absence of $\mathrm{CH}_{4}$ in the massive, high-velocity $-16 \mathrm{~km} \mathrm{~s}^{-1}$, warm $(T \simeq 270 \mathrm{~K})$ outflow seen in $\mathrm{CO}\left(X\left[\mathrm{CH}_{4}\right]<0.4 \times 10^{-6}\right)$.

These new results provide clues to the formation and evolutionary history of $\mathrm{CH}_{4}$, refining previous scenarios (Lacy et al. 1991; Boogert et al. 1998). In time-dependent pure gas-phase models, such as the "standard" dense cloud model of Lee et al. (1996) and the UMIST model of Millar et al. (1997), gas-phase $\mathrm{CH}_{4}$ abundances of a few $\times 10^{-6}$ are achieved after $10^{5} \mathrm{yr}$. Although this is comparable to the observed abundances, there are strong arguments against a gas-phase origin: the low gasstate/solid-state ratio, and the intimate mixture between $\mathrm{CH}_{4}$ and $\mathrm{H}_{2} \mathrm{O}$ in the ices in the envelope. For comparison, $\mathrm{CO}$, a typical gas-phase product, has an order-of-magnitude larger gas/solid phase ratio compared to $\mathrm{CH}_{4}$, even in the cold gas phase. $\mathrm{H}_{2} \mathrm{O}$, on the other hand, is formed by hydrogenation of oxygen on the grains at low temperatures (e.g., Jones \& Williams 1984), and a low gas/solid-state ratio is observed (Boonman \& van Dishoeck 2003). It is thus likely that $\mathrm{CH}_{4}$ was formed on grain surfaces by hydrogenation of atomic $\mathrm{C}$, at the same time that $\mathrm{H}_{2} \mathrm{O}$ was formed from atomic $\mathrm{O}$. The low observed $\mathrm{CH}_{4}$ abundance points to a formation time when most of the $\mathrm{C}$ was already locked up in CO (Boogert et al. 1998).

The gas-phase $\mathrm{CH}_{4}$ abundance in the -6 and $+8 \mathrm{~km} \mathrm{~s}^{-1}$ components is comparable to the solid-state abundance in the envelope. The $\mathrm{CH}_{4}$ may thus have been released from the grains in the inner regions near the protostar. Because $\mathrm{CH}_{4}$ is diluted in an $\mathrm{H}_{2} \mathrm{O}$-rich ice (Fig. 6), its sublimation temperature is close to that of $\mathrm{H}_{2} \mathrm{O}: 90 \mathrm{~K}$ (Tielens \& Whittet 1996; Hiraoka et al. 1998). The temperature of the $+8 \mathrm{~km} \mathrm{~s}^{-1}$ emission component is indeed well above $90 \mathrm{~K}$. The gas at $-6 \mathrm{~km}$ $\mathrm{s}^{-1}$, however, has a temperature of $55 \pm 15 \mathrm{~K}$ only. While in one massive protostar, $\mathrm{W} 33 \mathrm{~A}, \mathrm{CH}_{4}$ gas at $T=110 \mathrm{~K}$ was detected (Boogert et al. 1998), a lower temperature of $50 \mathrm{~K}$ was also measured in another line of sight, GL 7009S (Dartois et al. 1998). We speculate that the icy mantles did not thermally evaporate as a result of protostellar radiative heating, but by a mild shock that has briefly heated the grains above $90 \mathrm{~K}$. Subsequently, the gas has cooled radiatively or adiabatically as the inner NGC 7538 IRS 9 core expands outward with a (current) velocity of $6 \mathrm{~km} \mathrm{~s}^{-1}$.

Shocks may have had a profound impact on the higher velocity gas. $\mathrm{CH}_{4}$ is absent at velocities of $16 \mathrm{~km} \mathrm{~s}^{-1}$ and above, where warm $\mathrm{CO}(T=280 \mathrm{~K})$ is very abundant. After release from the grains, $\mathrm{CH}_{4}$ must have efficiently chemically reacted. The observed CO velocities up to $35 \mathrm{~km} \mathrm{~s}^{-1}$ point to the presence of nondestructive C-type shocks. These shocks have been well studied in the context of molecular chemistry. At initial densities of $10^{5} \mathrm{~cm}^{-3}$, shocks with velocities of $15-35 \mathrm{~km} \mathrm{~s}^{-1}$ have peak temperatures of 500-2500 K (Bergin et al. 1998). At such high temperatures, and at the low expected shock pressures, ${ }^{7} \mathrm{CH}_{4}$ is rapidly driven into CO (Prinn \& Fegley 1989). The occurrence of a $\mathrm{C}$ shock therefore explains the absence of $\mathrm{CH}_{4}$ in the outflow of NGC 7538 IRS 9. The importance of shock chemistry in highmass cores was deduced from low gas/solid $\mathrm{CO}_{2}$ abundance ratios as well (Charnley \& Kaufman 2000), and by the abundance ratios of S-bearing species (Hatchell \& Viti 2002). One distinct characteristic of shock chemistry is the formation of $\mathrm{H}_{2} \mathrm{O}$ at temperatures above $250 \mathrm{~K}$ (e.g., Bergin et al. 1998). $\mathrm{H}_{2} \mathrm{O}$ columns comparable to $\mathrm{CO}$ are expected, i.e., $2.8 \times 10^{18} \mathrm{~cm}^{-2}$

\footnotetext{
${ }^{7} \log _{10}(P)=-13$ bar, assuming a factor of 2 density enhancement at a maximum temperature of $2500 \mathrm{~K}$ for a $35 \mathrm{~km} \mathrm{~s}^{-1}$ shock (Kaufman \& Neufeld 1996; Bergin et al. 1998).
} 
for the NGC 7538 IRS 9 outflow (Table 4). This is a factor of 3.5 larger than the upper limit to the gas-phase $\mathrm{H}_{2} \mathrm{O}$ abundance derived from ISO SWS $6 \mu \mathrm{m}$ spectra (Boonman \& van Dishoeck 2003). High-resolution $6 \mu \mathrm{m}$ spectra are required to further investigate the presence of $\mathrm{H}_{2} \mathrm{O}$ in the line of sight of NGC 7538 IRS 9, the effect of blended emission and absorption components, and the relation to shocks and outflows.

\subsection{Nature of the Low-Velocity Warm Gas}

The elevated temperature of the low-velocity, $6-8 \mathrm{~km} \mathrm{~s}^{-1}$, absorption and emission components seen both in $\mathrm{CO}$ and $\mathrm{CH}_{4}$ locates this gas in the inner regions of the protostellar core. The present observations further constrain the origin of this material.

The area of the radiating physical body can be calculated by matching the ${ }^{12} \mathrm{CO}$ column density derived from the isotope ratio $(\S 3.2 .2)$ to the emission-line fluxes. The measured ${ }^{12} \mathrm{CO}$ line fluxes need to be corrected for continuum absorption by foreground material. We derive a $4.7 \mu \mathrm{m}$ continuum extinction magnitude of $A_{4.7}=1.8 \pm 0.6$ using the interstellar extinction law of Draine (2003) and an optical extinction $A_{V}$ of $60 \pm 20$. The latter is derived from the $A_{V} / N_{\mathrm{H}}$ relation of Bohlin et al. (1978), and the average line-of-sight hydrogen column density from this work (Table 4) and that from the silicate absorption band (Tielens et al. 1991). The extinction-corrected line fluxes are thus matched at a solid angle of $4.3 \times 10^{-14} \mathrm{sr}$. At the distance to NGC 7538 IRS 9 of $2.8 \mathrm{kpc}$ (Crampton et al. 1978) this corresponds to a radius of $70 \mathrm{AU}$. The similarity of $\mathrm{CO}$ and $\mathrm{CH}_{4}$ velocities and emission-line widths (Fig. 4c) indicates that both molecules are present in this same volume.

The $\mathrm{CO}$ and $\mathrm{CH}_{4}$ gas detected on these scales may well be related to the dense material that was inferred on scales of $\leq 300-600$ AU from unresolved $3 \mathrm{~mm}$ continuum interferometer images (van der Tak et al. 2000). This intense emission is unrelated to the envelope and instead may originate from a dense shell or circumstellar disk. The P Cygni-like line profiles in both $\mathrm{CO}$ and $\mathrm{CH}_{4}$ may suggest that an expanding envelope is the preferred geometry. In these line profiles the absorption component is redshifted by the shell expansion velocity, and the emission component is centered at the systemic velocity and is broadened by twice the expansion velocity. Mitchell et al. (1991) argue that the CO emission component is blueshifted because of the effect of deep absorption by foreground material at the systemic velocity. This hypothesis can be tested by the profiles of the highest $J$ transitions, which should not have foreground contributions. We find that even the lines from the highest rotational levels of $\mathrm{CO}$ are blueshifted by $+8 \mathrm{~km} \mathrm{~s}^{-1}$ (Fig. 4c). Furthermore, the $\mathrm{CH}_{4}$ emission lines peak at this same velocity and are also unresolved. Perhaps the NGC 7538 IRS 9 core moves within its envelope by $8 \mathrm{~km} \mathrm{~s}^{-1}$ and the observed peak of the emission line is the center of the P Cygni profile. In this scenario the shell expansion velocity is $14 \mathrm{~km} \mathrm{~s}^{-1}$, and an emission-line width of $28 \mathrm{~km} \mathrm{~s}^{-1}$ is expected. Such broad lines are inconsistent with the observations as well. We conclude that the line profiles are not true P Cygni type profiles and thus are inconsistent with a spherically symmetric expanding envelope.
It is unclear what the relation is between the $55 \mathrm{~K}$ absorbing gas seen at $-6 \mathrm{~km} \mathrm{~s}^{-1}$ and the $500 \mathrm{~K}$ emitting gas at $+8 \mathrm{~km} \mathrm{~s}^{-1}$. More detailed dynamical models of circumstellar disks, accretion flows, and outflows are required. The possibility of photon excitation needs to be considered as well. As noted in Mitchell et al. (1991) the similarity of CO absorption- and emission-line profiles between a number of massive protostars suggests that these lines trace a fundamental property of their inner cores. This is strengthened by the similarity of the $\mathrm{CH}_{4}$ line profiles.

\section{CONCLUSIONS AND FUTURE WORK}

Using high-resolution $L$ - and $M$-band spectroscopy, we have shown that the gas-phase $\mathrm{CH}_{4}$ abundance varies considerably in the line of sight of the massive protostar NGC 7538 IRS 9. This is a result of specific molecule formation and destruction processes: $\mathrm{CH}_{4}$ is formed on grain surfaces and subsequently evaporated from the grains in the inner regions of the protostar. Both evaporation by photon heating and by shocks likely have played roles. $\mathrm{CH}_{4}$ efficiently "burns" to $\mathrm{CO}$ in the highest velocity shocked regions. The gas-phase $\mathrm{CH}_{4} / \mathrm{CO}$ abundance ratio is thus a sensitive tracer of shock chemistry. High spectral resolution observations of other species, in particular $\mathrm{H}_{2} \mathrm{O}$ at $6 \mu \mathrm{m}$, are required to further test the importance and nature of shock chemistry in high-mass star-forming regions. The Stratospheric Observatory For Infrared Astronomy (SOFIA) will offer this opportunity in the near future (Lacy et al. 2002). Observations of the $3.32 \mu \mathrm{m} \mathrm{CH}_{4}$ stretch mode in more lines of sight require high radial velocities $\left(>30 \mathrm{~km} \mathrm{~s}^{-1}\right)$ or temperatures that populate the highest $J$ levels to avoid saturated telluric lines. In this respect, the $7.67 \mu \mathrm{m}$ bending mode may be more suited. Future observations of $\mathrm{CH}_{4}$ toward low-mass protostars are particularly exciting, as the $\mathrm{CH}_{4} / \mathrm{CO}$ ratio is a sensitive tracer of protoplanets. In the relatively low pressure solar nebula low $\mathrm{CH}_{4} / \mathrm{CO}$ abundances occur. The higher pressure in protoplanetary subnebulae, however, favors higher $\mathrm{CH}_{4} / \mathrm{CO}$ ratios (Prinn \& Fegley 1989). Variations of the $\mathrm{CH}_{4}$ abundance between solar system comets possibly trace such physical condition gradients in the early protoplanetary disk (Gibb et al. 2003).

The research of A. C. A. B. and G. A. B. is supported by the Spitzer Legacy Science program and by the Owens Valley Radio Observatory through NSF grant AST 02-28955. The authors wish to recognize and acknowledge the very significant cultural role and reverence that the summit of Mauna Kea has always had within the indigenous Hawaiian community. We are most fortunate to have the opportunity to conduct observations from this mountain. We thank Jan Cami for providing a copy of ISO SWS IA, Fred Lahuis for an independent reduction, Tim Brooke for suggesting the possible ethane identification, and Yuk Yung for help with the wavelength calibration. Suggestions by an anonymous referee have led to an improved presentation of the data.

\section{REFERENCES}

Alexander, R. D., Casali, M. M., André, P., Persi, P., \& Eiroa, C. 2003, A\&A, 401,613

Allamandola, L. J., Sandford, S. A., Tielens, A. G. G. M., \& Herbst, T. M. 1992, ApJ, 399, 134

Bergin, E. A., Neufeld, D. A., \& Melnick, G. J. 1998, ApJ, 499, 777

Bohlin, R. C., Savage, B. D., \& Drake, J. F. 1978, ApJ, 224, 132

Boogert, A. C. A., Blake, G. A., \& Tielens, A. G. G. M. 2002, ApJ, 577, 271
Boogert, A. C. A., Helmich, F. P., van Dishoeck, E. F., Schutte, W. A., Tielens, A. G. G. M., \& Whittet, D. C. B. 1998, A\&A, 336, 352

Boogert, A. C. A., Schutte, W. A., Helmich, F. P., Tielens, A. G. G. M., \& Wooden, D. H. 1997, A\&A, 317, 929

Boogert, A. C. A., et al. 1996, A\&A, 315, L377

. 2000, A\&A, 353, 349 2004, ApJS, 154, 359 
Boonman, A. M. S., \& van Dishoeck, E. F. 2003, A\&A, 403, 1003 Boudin, N., Schutte, W. A., \& Greenberg, J. M. 1998, A\&A, 331, 749

Brooke, T. Y., Sellgren, K., \& Geballe, T. R. 1999, ApJ, 517, 883

Brooke, T. Y., Sellgren, K., \& Smith, R. G. 1996, ApJ, 459, 209

Brown, L. R., et al. 2003, J. Quant. Spectrosc. Radiat. Transfer, 82, 219

Brown, P. D., Charnley, S. B., \& Millar, T. J. 1988, MNRAS, 231, 409

Ceccarelli, C., Loinard, L., Castets, A., Tielens, A. G. G. M., Caux, E., Lefloch, B., \& Vastel, C. 2001, A\&A, 372, 998

Charnley, S. B., \& Kaufman, M. J. 2000, ApJ, 529, L111

Chiar, J. E., Tielens, A. G. G. M., Whittet, D. C. B., Schutte, W. A., Boogert, A. C. A., Lutz, D., van Dishoeck, E. F., \& Bernstein, M. P. 2000, ApJ, 537, 749 Crampton, D., Georgelin, Y. M., \& Georgelin, Y. P. 1978, A\&A, 66, 1

Dartois, E., D’Hendecourt, L., Boulanger, F., Jourdain de Muizon, M., Breitfellner, M., Puget, J.-L., \& Habing, H. J. 1998, A\&A, 331, 651

Dartois, E., D’Hendecourt, L., Thi, W., Pontoppidan, K. M., \& van Dishoeck, E. F. 2002, A\&A, 394, 1057

Dartois, E., Schutte, W., Geballe, T. R., Demyk, K., Ehrenfreund, P., \& D'Hendecourt, L. 1999, A\&A, 342, L32

de Graauw, T., et al. 1996, A\&A, 315, L49

Draine, B. T. 2003, ARA\&A, 41, 241

Fischer, J., Gamache, R. R., Goldman, A., Rothman, L. S., \& Perrin, A. 2003, J. Quant. Spectrosc. Radiat. Transfer, 82, 401

Genzel, R. 1992, in The Galactic Interstellar Medium, ed. D. Pfenniger \& P. Bartholdi (Berlin: Springer), 275

Gerakines, P. A., et al. 1999, ApJ, 522, 357

Gibb, E. L., Mumma, M. J., dello Russo, N., Disanti, M. A., \& Magee-Sauer, K. 2003, Icarus, 165, 391

Gürtler, J., Klaas, U., Henning, T., Ábrahám, P., Lemke, D., Schreyer, K., \& Lehmann, K. 2002, A\&A, 390, 1075

Hatchell, J., \& Viti, S. 2002, A\&A, 381, L33

Hiraoka, K., Miyagoshi, T., Takayama, T., Yamamoto, K., \& Kihara, Y. 1998, ApJ, 498, 710

Jones, A. P., \& Williams, D. A. 1984, MNRAS, 209, 955

Kaufman, M. J., \& Neufeld, D. A. 1996, ApJ, 456, 250

Kessler, M. F., et al. 1996, A\&A, 315, L27

Knacke, R. F., Noll, K. S., Geballe, T. R., \& Tokunaga, A. T. 1985, ApJ, 298, L67

Lacy, J. H., Carr, J. S., Evans, N. J., Baas, F., Achtermann, J. M., \& Arens, J. F. 1991, ApJ, 376, 556

Lacy, J. H., Knacke, R., Geballe, T. R., \& Tokunaga, A. T. 1994, ApJ, 428, L69
Lacy, J. H., Richter, M. J., Greathouse, T. K., Jaffe, D. T., \& Zhu, Q. 2002, PASP, 114, 153

Lee, H.-H., Bettens, R. P. A., \& Herbst, E. 1996, A\&AS, 119, 111

Lide, D. R., 1994, CRC Handbook of Chemistry and Physics (75th ed.; Boca Raton: CRC)

Markwick, A. J., Millar, T. J., \& Charnley, S. B. 2000, ApJ, 535, 256

McLean, I. S., Becklin, E. E., Bendiksen, O., Brims, G., \& Canfield, J. 1998, Proc. SPIE, 3354, 566

Millar, T. J., Farquhar, P. R. A., \& Willacy, K. 1997, A\&AS, 121, 139

Mitchell, G. F., Maillard, J.-P., Allen, M., Beer, R., \& Belcourt, K. 1990, ApJ, 363,554

Mitchell, G. F., Maillard, J.-P., \& Hasegawa, T. I. 1991, ApJ, 371, 342

Noriega-Crespo, A., et al. 2004, ApJS, 154, 352

Pendleton, Y. J., Sandford, S. A., Allamandola, L. J., Tielens, A. G. G. M., \& Sellgren, K. 1994, ApJ, 437, 683

Pontoppidan, K. M., Dartois, E., van Dishoeck, E. F., Thi, W.-F., \& D’Hendecourt, L. 2003, A\&A, 404, L17

Prinn, R. G., \& Fegley, B. 1989, in Origin and Evolution of Planetary and Satellite Atmospheres, ed. S. K. Atreya, J. B. Pollack, \& M. S. Matthews (Tucson: Univ. Arizona Press), 78

Rothman, L. S., et al. 2003, J. Quant. Spectrosc. Radiat. Transfer, 82, 5

Sandford, S. A., Allamandola, L. J., Tielens, A. G. G. M., Sellgren, K., Tapia, M., \& Pendleton, Y. 1991, ApJ, 371, 607

Schutte, W. A., Gerakines, P. A., Geballe, T. R., van Dishoeck, E. F., \& Greenberg, J. M. 1996, A\&A, 309, 633

Spitzer, L. 1978, Physical Processes in the Interstellar Medium (New York: Wiley)

Spoon, H. W. W., Keane, J. V., Tielens, A. G. G. M., Lutz, D., \& Moorwood, A. F. M. 2001, A\&A, 365, L353

Tielens, A. G. G. M., Tokunaga, A. T., Geballe, T. R., \& Baas, F. 1991, ApJ, 381,181

Tielens, A. G. G. M., \& Whittet, D. C. B. 1996, in IAU Symp. 178, Molecules in Astrophysics: Probes \& Processes, ed. E. F. van Dishoeck (Dordrecht: Kluwer), 45

van der Tak, F. F. S., van Dishoeck, E. F., Evans, N. J., \& Blake, G. A. 2000, ApJ, 537, 283

White, G. J., et al. 2000, A\&A, 364, 741

Whittet, D. C. B., et al. 1996, A\&A, 315, L357

Willner, S. P., et al. 1982, ApJ, 253, 174

Wilson, T. L., \& Rood, R. T. 1994, ARA\&A, 32, 191 